\title{
Elucidation of the metabolic fate of glucose in Trichoderma ressei using EST analysis and cDNA microarray: a step towards metabolic engineering of the regulatory networks for anaerobic metabolism for glucose
}

\author{
Hamza El-Dorry ${ }^{1,2,3}$
}

\author{
Address: ${ }^{1}$ Biotechnology Graduate Program, American University in Cairo, Cairo, Egypt, ${ }^{2}$ Biology Department, American University in Cairo, \\ Cairo, Egypt and ${ }^{3}$ YJ-Science and Technology Research center, American University in Cairo, Cairo, Egypt \\ from 2009 American University in Cairo Research Conference \\ Cairo, Egypt. 5 April 2009
}

Published: I July 2009

BMC Proceedings 2009, 3(Suppl 3):O2

This abstract is available from: http://www.biomedcentral.com/I753-656I/3/S3/O2

(c) 2009 El-Dorry; licensee BioMed Central Ltd.

Glycolysis, the tricarboxylic acid cyle (TCA) and the electron transport chain are part of the metabolic systems of the cell required for the production of energy in the form of ATP from glucose and are common to all eukaryotic microorganisms. Different regulatory networks have developed during evolution in different microorganisms to control these networks to produce ATP by different pathways. For example, in the presence of high concentration of glucose the yeast Saccharomyces cerevisiae produces energy by anaerobic metabolism accumulating ethanol as the end product of the process. The cellulolytic fungus Trichoderma reesei, however, produces energy from glucose by aerobic metabolism. We have recently addressed this difference using EST analysis and cDNA microarrays. We show that in $T$. reesei expression of the networks of genes encoding the enzymes of glycolysis, the TCA cycle and the proteins of the electron transport chain is programmed in a way that favours the oxidation of pyruvate via the TCA cycle rather than its reduction to acetaldehyde and then ethanol by fermentation. In addition, our results indicate that the metabolic step required to regenerate NAD+ is regulated differently in S. cerevisiae and T. reesei, in Trichoderma preventing the regeneration of this pivotal product that is required for anaerobic metabolism. Thus, in $T$. reesei, in contrast to $S$. cerevisiae, aerobic metabolism prevails in the presence of high levels of glucose. To turn this preferentially respiratory microorganism into a fermenter, we are replacing the promoters of critical genes whose products control the direction of flow of the primary metabolites toward respiratory metabolism, with tightly glucose-repressible promoter. We expect that these promoter-engineering will channel the metabolites toward anaerobic metabolism of glucose and turn this preferentially respiratory microorganism into a fermenter-a step that will be required if $T$. reesei is to be used for the efficient production of ethanol fuel from cellulose biomass. 